\title{
Diversity and Distribution of Potential Pathogens and Antibiotic Resistance Genes in Anthropogenic Disturbances Aquatic Environment and Their Relationship with Microbial Indicators
}

\author{
Xiang-Long Zhao ( $2070611418 @ q q . c p m$ ) \\ Anhui Agricultural University \\ Zhao Qi \\ Anhui Agricultural University \\ Hao Huang \\ Anhui Agricultural University \\ Jian Tu \\ Anhui Agricultural University \\ Xiang-Jun Song \\ Anhui Agricultural University \\ Ke-Zong Qi ( $\nabla$ qkz@ahau.edu.cn ) \\ Anhui Agricultural University \\ Yin Shao \\ Anhui Agricultural University
}

\section{Research Article}

Keywords: Next-generation sequencing, Opportunistic pathogens, Antimicrobial resistance, Aquatic microbiological risk

Posted Date: July 19th, 2021

DOl: https://doi.org/10.21203/rs.3.rs-601610/v1

License: (9) This work is licensed under a Creative Commons Attribution 4.0 International License. Read Full License 


\section{Abstract}

Microbial indicators are often used as alternative indicators of microbial safety in water. However, information regarding the correlation between microbial indicators and ecotoxicological factors such as potential pathogens and antibiotic resistance genes (ARGs) in anthropogenically impacted waters remains highly limited. Combining $16 \mathrm{~S}$ rRNA and metagenomic sequencing data, we investigated the composition of bacterial community and potential pathogens, ARGs diversity, ARGs host and horizontal gene transfer (HGT) potential in water samples under the influence of different exogenous pollutants in Chaohu Lake basin. The water body that receives a large amount of domestic sewage showed a significant decrease in microbial diversity and a significant enrichment of potential pathogens. A total of 14 main types and 461 subtypes of ARGs were detected in all samples, dominated by multidrug resistance (MDR) efflux pump (53.6\%), aminoglycoside (6.0\%), fluoroquinolone (5.8\%) and polymyxin (5.46\%). Host-tracking analysis showed that Escherichia coli and Bacteroides graminisolvens carried a wealth of ARG subtypes. Correlation analysis showed that potential pathogens and some ARG subtypes such as dfrE, sul2, PmrE exhibits significant correlation with indicator bacteria. Overall, next-generation sequencing (NGS) has the ability to conduct preliminary surveys of environmental samples to access potential health risks, thus providing ideas for water resources management.

\section{Introduction}

Aquatic pathogenic microorganisms have become one of the major threats to public health and have attracted worldwide attention (Schmeller et al. 2018). Waterborne infectious diseases, such as diarrhoea, have historically been associated with poor sanitation, inappropriate hygiene and the use of unsafe water. There are 4 billion cases of diarrhoea reported among the world each year, of which 2.2 million are fatal, the main victims being children under 5 years of age (Ahmed et al. 2020), and a variety of aquatic pathogens have been shown to have the potential to cause waterborne, healthcare-associated infections. Pathogens in the aquatic environment can be divided into intestinal and aquatic / environmental pathogens (Cui et al. 2019), in which the main sources of intestinal pathogens are human and animal feces (Byappanahalli et al. 2012). With the continuous expansion of China's livestock and poultry industry, sewage production gradually increased, continue to exert harmful microbial stress on the environment. According to the 2015 China Environmental Statistics Annual Report (http://www.mee.gov.cn/hjzl/sthjzk/sthjtjnb/), the chemical oxygen demand (COD) emissions of China's livestock and poultry breeding industry reached 10.155 million tons in 2015 , and a considerable amount of sewage was discharged into rivers. In addition, due to the lack of sewage treatment facilities in smallscalefarms, the discharge of waste sewage without proper treatment, untreated fecal sewage may become a major source of harmful microorganisms in the environment, so it is important for water fecal contamination and monitoring of potential pathogens.

The detection and counting of indicator microorganisms has been the gold standard for evaluating the safety of aquatic microorganisms in the past few decades (Figueras and Borrego 2010). However, the relationship between indicator microorganisms and potential pathogens in the environment is still 
unclear, most studies have not reported the correlation between them, or the relationship between them is not statistically significant (Korajkic et al. 2018), and few studies have focused on the co-occurence relationship between indicator microorganisms and drug resistance genes in the environment. Furthermore, although coliforms has been established as sanitary standards of the safety of fresh water, it is shocking that the problem of multiple drug resistance was on the rise (Mishra et al. 2018). Coliforms are extremely receptive and altruistic to horizontal gene transfer in nature, so it is easy to become a hot spot to accept and spread antibiotic resistance genes in the environment (Triggiano et al. 2020).

Subsequently, a method of combining FIB with microbial source tracking (MST) indicators was developed to enhance the ability to identify sources of contamination (Duan et al. 2016) by applying techniques such as culture or quantitative PCR (qPCR), and this method have the ability to assess the potential health risks of water bodies to a certain extent (Purnell et al. 2020). However, while qPCR has the ability to quantify fecal contamination and pathogenic bacteria in water, the low throughput of such methods does not provide a comprehensive assessment of the potential health risks of water bodies, as most potential pathogens in ambient water have high diversity and low concentrations (Harwood et al.

2014). Furthermore, the discharge of livestock manure not only brings a large number of potential pathogens, but also may cause a high risk of antimicrobial resistance (AMR), the increase of AMR in water poses a risk to human health and increases the possibility of skin contact or ingestion of potential drug resistance and pathogenic bacteria (Neher et al. 2020). The spread of antibiotic resistance genes (ARGs) and their acquisition by clini-cally relevant pathogens have made serious problems with human and animal health. However, the monitoring of antibiotic drug resistance genes is still not part of the water quality monitoring frameworks, and it is still necessary to better understand the occurrence and characteristics of Args in the environment to control their propagation and evolution (Bush et al. 2011).

The development of NGS has provided new ideas for water pollution assessment, and most members of the water body bacterial community can be identified by targeting traditional 16S genes (Yue et al. 2020), this method has the ability to identify contaminants from complex sources in water bodies because NGS sequencing can use multiple markers as a signature to avoid uncertainty caused by a single marker. In addition, the public health risk of contaminated water can be assessed by screening sequences that are highly associated with pathogens (Lu et al. 2015), but the reference pathogen database used in this method is a key factor, which will affect the accuracy and diversity of detection. In short, despite many limitations, NGS-based microbial community analysis is of great significance for preliminary investigation of water samples to assess public health risks associated with fecal contamination or pathogens. High-throughput sequencing (HTS) has been widely used to investigate the quantitative occurrence, abundance and diversity of args in the environment (Chen et al. 2019). With regard to traditional molecular techniques, metagenomics analysis can used for broad-spectrum scanning of ARGs in environmental samples, thus obtaining the overall abundance and occurrence information (Yang et al. 2013). 
With more than 9.1 million people living in the Chaohu River basin, as a fast-growing economic zone with a variety of important industries, the continuous and rapid development of industrial and agricultural development over the years has brought serious pollution and eutrophication problems in downstream areas (Yang et al. 2016). Previous studies have focused on the denutrification and antibiotic contamination of the water bodies of the Chaohu watershed, which proved that Chaohu watershed was polluted by agricultural non-point sources and urban domestic sewage (Yang et al. 2020), In addition, eutrophication pollution led to the decrease of bacterial diversity in the water body of Chaohu Lake (Zhang et al. 2020). However, these studies have not paid attention to the distribution of potential pathogenic bacteria and ARGs in Chaohu Basin, which is extremely important for public health. To address this knowledge gap, we combined 16S rRNA sequencing and metagenome-assembled genome analysis to determine (1) The differences in bacterial community composition, including the abundance and diversity of potential pathogens. (2) Categories related to antibiotic resistomes (ARs), including the abundance, diversity, potential host and gene transfer potential by mobile genetic elements, of ARGs, including plasmids, phages and transposons, using assembled contigs to predict ARGs and MGEs, so as to obtain more accurate results (Chen et al. 2019). (3) Explore the relationship between indicator microorganisms and potential pathogens and ARGs based on ARG-carrying contigs (ACCs) and Network analysis.

\section{Material And Methods}

\section{Collection of River Water Samples}

In August 2019, a total of 36 water samples were collected from three main tributaries of Chaohu Basin and an agricultural non-point source pollution monitoring station. Among them, Hangbu (HR)-Fengle (FR) River is the largest inflow river in Chaohu Lake, accounting for $65.1 \%$ of the total runoff, with a watershed area of $4414 \mathrm{~km}^{2}$. The tributary is mainly polluted by agricultural non-point source pollution and domestic sewage runoff on both sides of the river, while the other tributary river Paihe (XR) mainly receives agricultural source sewage. The sampling sites also include the influent (D) and effulent (E) of a wastewater treatment plant (WWTP) which mainly treats wastewater from rural areas where livestock were present and the ecological interception ditch (A) downstream of the effulent (Fig. S1). Specific aquatic plants are planted in ecological interception ditches, which can alleviate the flow velocity, promote the precipitation of sediment particles, and absorb and intercept $\mathrm{N}$ and $\mathrm{P}$ in the water (Di et al. 2016). The water samples were collected with $500 \mathrm{ml}$ aseptic plastic bottles, stored on ice and transported to the laboratory for treatment within 8 hours. Then, $500 \mathrm{~mL}$ of each samples were filtered through a $0.22 \mu \mathrm{m}$ polycarbonate membrane filter and stored at $-20^{\circ}$ until DNA extraction.

\section{DNA extraction and high-throughput sequencing}

DNA from different samples was extracted using the E.Z.N.A. ®Stool DNA Kit (D4015, Omega, Inc., USA) according to manufacturer's instructions. The reagent which was designed to uncover DNA from trace amounts of sample has been shown to be effective for the preparation of DNA of most bacteria. Nuclear- 
free water was used for blank. The total DNA was eluted in $50 \mu \mathrm{L}$ of Elution buffer and stored at $-80^{\circ} \mathrm{C}$ until measurement in the PCR. After detection of DNA density, 36 DNA samples ( 6 per site) were used for 16S rRNA sequencing, one sample of each site was chosen for pairedend shotgun metagenomic metagenomic sequencing (see Text S1 for details), bioinformatic analyses of 16S rRNA amplicon sequencing can be found in Text S2.

\section{Assessment of potential pathogenic bacteria abundance by 16S rRNA sequencing}

In order to identify the potential pathogens in the sequencing data, a list of potential pathogens was established, including 159 genera (Table S2), according to the VFDB website (http://www.mgc.ac.cn/VFs/) and the Pathosystems Resource Integration Center (PATRIC) (Wattam et al. 2014), and reference lists from other studies (Cui et al. 2019; Fang et al. 2018; Liu et al. 2018; Li et al. 2020), each genus was then searched on the American Biological Safety Association (ABSA) (https://my.absa.org/tiki-index.php?page=Riskgroups) record the species considered bio-safety level 2 or 3. The reference 16S rRNA sequences of potential pathogen species were extracted from the LPSN - List of Prokaryotic names with Standing in Nomenclature (https://www.bacterio.net/) and GeneBank (https://www.ncbi.nlm.nih.gov/genbank/). Finally, a reference potential pathogen database was constructed, consisting of 51 genera and 444 species, can be found in (https://github.com/Titanium1024/Reference-sequence-for-Potential-Pathogens.git). Unique reads classified to the genera in the reference database of human pathogenic bacteria were screened out and aligned with the representative sequences of pathogenic species within the same genera using BLAST.

Nucleotide sequences of amplicons were aligned with the reference potential pathogen database using BLASTN with the identity $\geq 97 \%$ and coverage $>90 \%$, the relative abundance of potential pathogens was calculated by dividing the abundance of the feature values corresponding to the sequences of potential pathogens by the abundance of the total feature values of the samples.

\section{Gene prediction, taxonomy, and functional annotation}

Open reading frames (ORFs) from each assembled contig were predicted using MetaGene (Noguchi et al. 2006) (http://metagene.cb.k.u-tokyo.ac.jp/). The predicted ORFs with length being or over $100 \mathrm{bp}$ were retrieved and translated into amino acid sequences using the NCBI translation table (http://www.ncbi.nlm.nih.gov/Taxonomy/taxonomyhome.html/index.cgi?chapter=tgencodes\#SG1). All predicted genes with a $95 \%$ sequence identity (90\% coverage) were clustered using CD-HIT (Fu et al. 2012) (http://www.bioinformatics.org/cd-hit/), the longest sequences from each cluster were selected as representative sequences to construct non-redundant gene catalog. Reads after quality control were mapped to the representative sequences with 95\% identity using SOAPaligner (Li et al. 2008) (http://soap.genomics.org.cn/) , and gene abundance in each sample were evaluated based on reads per kilobase per million mapped reads (RPKM). Representative sequences of non-redundant gene catalog were aligned to NCBI NR database with e-value cutoff of $1 e^{-5}$ using BLASTP (Version 2.2.28+, http://blast.ncbi.nlm.nih.gov/Blast.cgi) (Altschul 1997) for taxonomic annotations. Cluster of 
orthologous groups of proteins (COG) annotation for the representative sequences was performed using BLASTP against eggNOG database (Tatusov et al. 2003; Li et al. 2008) (Version4.5) with an e-value cutoff of 1e-5. The KEGG annotation was conducted using BLASTP (Version 2.2.28+) against the Kyoto Encyclopedia of Genes and Genomes database (Xie et al. 2011) (http://www.genome.jp/keeg/) with an evalue cutoff of $1 \mathrm{e}^{-5}$. With regard to the horizontal gene transfer potential, prophages and plasmids were annotated by comparison with the ACLAME database (v0.4), the ISfinder database was used to determine the insertion sequences. Antibiotic resistance annotation was conducted using BLASTP search (Version 2.2.28+) against CARD database (http://arpcard.mcmaster.ca) (Jia et al. 2017) with an e-value cutoff of $1 \mathrm{e}-5$.

The correlation matrix between ARGs and water quality indicator bacteria was calculated based on the significant correlation of $p<0.01$ and Pearson's $r>0.80$, the relationship matrix between highly mobile ARGs and water quality indicator bacteria was calculated based on the significant correlation of $p<0.05$ and Pearson's $r>0.50$. $P$ value were generated via R-function "rcorr" (Hmisc package), $P$ values were then adjusted with Benjamini-Hochberg method to reduce false positive results (Ma et al. 2017). The network analysis was performed in $\mathrm{R}$ (version 3.6.1) environment using igraph (1.2.6), and was visualized by Gephi 0.9.2 (Bastian, Heymann, and Jacomy 2009).

\section{Statistical Analysis}

Kruskal-Wallis test and Wilcoxon test were used for global comparison and paired comparison to test the difference of the diversity index among different samples $(p<0.05)$. SPSS version 2.0 (IBM Corporation, Armonk, USA) was used for statistical analysis, and the significant value was set to $p<0.05$.

\section{Results}

\section{Differences in composition and diversity of bacteria communities, focusing on potential pathogens and indicator bacteria}

A total of $1,586,824$ high-quality reads were obtained after quality filtering, and the average number of sequences per sample was 44,078 , ranging from 24,690 to 108,652. A total of 29603 feature values were obtained through QIIME2 processing, with an average of 822, and sample coverage from $98.72 \%$ to 99.97\% (Table S1). The rarefaction curve (Fig. S2) based on species richness index indicates that the sequencing profundity was depth. Compared with other sampling sites, group D showed a significant decrease in chao1, shannon, simposn diversity index and observed otus (Kruskal-Walls, $p<0.01$ ) (Fig. S3). Furthermore, the unifrac distance was evaluated by Principal co-ordinates Analysis (PCoA) to reveal the differences in bacterial community composition among all samples (Fig. 1a), a significant segregation between two groups was observed. Differences in bacterial community were characterized using LefSe method (Fig. S4). All samples were divided into river group (XR, HR and FR) and wastewater group (D, E and A). The results showed that 10 phyla, 17 classes, 33 orders, 46 families, and 62 genera with significant spatial variation when a LDA threshold of 3 was used, at the phylum level, Actinobacteria, 
Cyanobacteria, Chloroflexi, Planctomycetes and Gemmatimonadetes are significantly enriched in river water, while Firmicutes, Epsilonbacteraeota, Synergistetes, Proteobacteria, Patescibacteria and Bacteroidales (from order to species) dominated in wastewater (Fig. 1b).

After the alignment of self-built potential pathogen database, a total of 60 potentially pathogenic species (Table S3) were found in all samples, which were clustered into 34 genera containing potential pathogenic species (Fig. 2). Group D harbored the highest relative abundance of potential pathogens $(0.0624 \%$ on average), followed by FR $(0.0347 \%)$. Pseudomonas, Mycobacterium, Comamonas and Aeromonas were the most widely distributed potential pathogens genera in this study. Among them, the relative abundance of Pseudomonas and Mycobacterium in group A was significantly higher than other groups ( $p<0.05$ or $p<0.001$, Wilcoxon), the relative abundance of Aeromonas in group E was significantly higher than that in group A $(p<0.01), \mathrm{D}(p<0.01)$ and $\mathrm{HR}(p<0.01)$, simultaneously, Comamona showed the highest relative abundance in group E. $(p<0.05$ or $p<0.001)$, Klebsiella was detected in 4 sites of FR and one site of HR, but not in wastewater group. It is worth noting that Bacillus was detected in 11 samples from $A$ and $E$, but not at $D$, indicating that the potential pathogens from this genus originated from the sewage treatment plant rather than other pollutants. Comamonas terrigena and Pseudomonas alcaligenes were the most frequently detected potential pathogen species (Fig. 2, Table S3). The highest density of Pseudomonas alcaligenes was observed in A3 and A5, Comamonas terrigena was detected in all sites except D5 and D6, and it's relative abundance increased after sewage treatment.

According to the Guidelines for Drinking-water Quality (WHO), Escherichia, Citrobacter, Klebsiella and Enterobacter, Serratia and Hafnia were choosed as indicator microorganisms, which contained most of the members of the total coliforms (TC), aerobic and facultatively anaerobic, Gram-negative, non-sporeforming bacilli capable, can survive in water environment. Moreover, the genus Bacteroides, Prevotella, Faecalibacterium, Helicobacter and the species Clostridium perfringens were classified as FIB, both of which was the member of the human and animal gut microbial community, can hardly reproduce in the environment, widely used as microbial source tracking (MST) (Shin et al. 2019; Duan et al. 2016; Ahmed et al. 2016). In addition, Enterococcus was classified as FIB group, which was recommended as indicators of fecal pathogens in drinking water standards. Specifically, here we classified Escherichia coli as a member of TC rather than FIB, because the source of Escherichia coli cannot be well identified through amplicon next-generation sequencing (Devane et al. 2020). Significant differences in the distribution of fecal indicator bacteria among all groups were observed (Fig. 2). Group D harbored the highest proportions of indicator bacteria $(1.31 \%$ on average, $p<0.001)$, dominated by Bacteroides $(0.78 \%$ on average) and Escherichia Shigella ( $0.34 \%$ on average), followed by FR ( $1.04 \%$ on average, $p<0.001)$, predominated by Klebsiella ( $0.86 \%$ on average) and Enterobacter $(0.13 \%$ on average). Compared with group $D$, the relative abundance of indicator bacteria decreased significantly in group $E$ and group $A$ ( $p<$ $0.001)$, the relative abundance of TC in the XR group was higher than that in FR and XR ( $p<0.05$ or $p<$ $0.01)$. The results of metagenomic sequencing showed a trend similar to that of $16 \mathrm{~S}$ rRNA sequencing (Fig. S4). 


\section{Diversity, occurrence and potential hosts of ARGs}

14 major types and 461 subtypes of ARGs were detected from the CARD database, and the number of ARGs in each sample ranged from 274 to 420,221 shared ARGs were detected in all samples. The distribution pattern of ARGs was significantly different among all samples (Kruskal-Wallis, $p<0.001$ ), D was significantly higher than that of other samples $(p<0.001)$, while A had the lowest ARGs relative abundance ( $p<0.01$ or $p<0.001$, Wilcoxon). The most prominent ARG types were multidrug resistance (MDR) efflux pump (53.6\%), aminoglycoside (6.0\%), fluoroquinolone (5.8\%), polymyxin $(5.46 \%)$ and isoniazid (4.5\%) (Fig. 3) there was no significant difference in ARGs abundance between XR, FR and HR. Furthermore, the abundance of trimethoprim $(p<0.001)$, polymyxin $(p<0.01)$, lipopeptide $(p<0.01)$ resistance gene in the wastewater group was significantly higher than that in the river water group, while the abundance of ethambutol resistance gene $(p<0.01)$ in the river water group was significantly higher than that in the wastewater group, but its relative abundance was relatively low $(0.03 \%$ of the total ARGs abundance).

It is worth noting that a higher relative abundance of polymyxin resistance gene such as PmrA, PmrB, PmrC, PmrE, PmrF was found at site D, but a significant decrease was observed at E and A. MCR-1 was detected in all samples except $\mathrm{E}$, but the relative abundance was relatively low. The relative abundance of some subtypes of glycopeptide resistance gene, such as vanTG, vanL, did not decrease after sewage treatment, while the relative abundance of vanO, vanB, vanKI, vanC, vanRO, vanF, vanSG, vanRG, vanA increased significantly after sewage treatment. In addition, except for some low-abundance ARG such as streptogramin $(0.07 \%)$, elfamycin $(0.04 \%)$, most of the ARG levels at WWTP effluent were significantly lower than those at the influent $(p<0.001)$. Similarly, a significant decrease in the total abundance of antibiotic resistance genes was observed in the ecological interception ditch downstream of the sewage outlet. Furthermore, we aligned the AACs against the NR database (e-value $\leq 1 \mathrm{e}-5$ ) using BLAST analyse, whereas $71.37 \%$ of ARG-Carrying contigs can be annotated at genus level. Escherichia coli, Bacteroides graminisolvens, Enterobacter cloacae were the main ARGs carrier, which was annotated to 54, 39 and 10 ARGs subtypes respectively, and these species are mainly distributed in site D (WWTP influent).

It is worth noting that 20 species were simultaneously annotated as potential pathogen, Arcobacter butzleri and Stenotrophomonas maltophilia carried 36 ARG subtypes, Pseudomonas alcaligenes and Klebsiella pneumoniae carried 17 and 4 drug resistance gene subtypes respectively. The most abundant type of ARGs carried by potential pathogens was efflux pump, followed by fluoroquinolone resistance gene (Table S5), the highest relative abundance of ARGs carried by potential pathogens was registered in site D, followed by FR. Furthermore, the main hosts of polymyxin are Achromobacter and Burkholderia, which account for $28.5 \%$ and $25.5 \%$ of the total, respectively.

\section{Horizontal gene transfer potential of ARGs}

The predicted ORFs was translated into amino acid sequence, and then alignment with MGE database by BLASTp analysis to evaluate the level of mobile genetic elements (MGEs). Alignment results showed that there were a total of 9294 Contigs with movable elements in all samples (WithE-value $\leq 10 \%$ similarity $\geq$ 
$80 \%$ focus coverage $\geq 70 \%$ ), of which the most abundant type of MGEs was plasmid. The highest relative abundance of MGEs was observed at site D, followed by FR. Meanwhile, a significant decrease of MGEs abundance was observed at point E compared with point $D$, the prominent MGE type found in site D was plasmid, while the dominant type in FR was IS (Fig. 4).Consistent with the change trend of ARGs, a significant decrease in MGEs level was observed after sewage treatment.

In order to identify the mobile ARGs, we matched the Contigs annotated as ARGs with the annotated Contigs carrying MGEs (the ARG carryings Contigs contains at least one MGE), 80, 166, 120, 90,94 and 116 Contigs carrying both ARG and MGE were detected in $A, D, E, X R, F R$ and XR, respectively, with the dominate type of plasmid (Table S5). Subsequently, a total of 15 ARGs were found to coexist with MGEs, and the main types were elfamycin, efflux pump, aminoglycoside, iSoniazid and sulfonamide resistance gene. We further performed taxonomic annotations using BLAST analysis on these ARG and MGE carrying contigs to determining the taxonomic origin. $84.94 \%$ of the Contigs that could be annotated to the genus level were Thauera (23.75\%), Pseudomonas (10.62\%), Escherichia (7.22\%), and Acinetobacter (6.48\%) (Fig. 5).

There were differences in dominant types of drug resistance among mobile ARGs carriers. For instance, Thauera mainly carried elfamycin resistance gene (97.96\%), with Escherichia coli EF-tu mutants conferring resistance to Enacyloxin IIA as the dominant subtype. Pseudomonas mainly carried sulfonamide resistance gene (52.23\%), followed by Efflux pump (29.20\%), Escherichia mainly carried aminoglycoside resistance gene (51.78\%), followed by elfamycin resistance gene $(22.87 \%)$ and Acinetobacter mainly carried sulfonamide resistance gene (73.43\%) (Fig. 5).

\section{Correlation analysis of indicator bacteria and potential pathogens and ARGs subtypes}

Pearson correlation analysis showed that the relative abundance of potential pathogens was significantly correlated with FIB, while TC was not significant, indicating that FIB was forceful to predict potential pathogens (Fig. 6). Network analysis approach was used to discern the co-occurrence patterns among TC and FIB communities and ARGs. The nodes are colored according to different bacterial taxa and ARG subtypes. The co-occurrence network consists of 233 nodes and 1714 edges. In this network, the average weighting degree is 14.804 , the network diameter is 14 , the graph density is 0.063 , the modularization index is 0.666 , the connection components are 13 , and the average network distance between all paired nodes is 13 . That is, the average path length is 5.832 and the average clustering coefficient is 0.701 (Fig. 7). In general, 20 ARG subtypes showed significant correlation with TC, while 10 ARGs showed significant correlation with FIB. TC and FIB members exhibited close correlations, dfrE, sul2, mexD, PmrE, oprA and FIB and TC community were observed as inter-type co-occurrence correlations. Specifically, dfrE, sul2, PmrE, which encodes trimethoprim, sulfonamide and polymyxin resistance, exhibits a significant correlation with 3 of FIB and 3 of TC members, while oprA and four kinds of FIB and one kind of TC members showed closely related.In particular, Klebsiella was the indicator bacteria most closely related to ARGs, and it was positively associated with 14 ARG subtypes of which 10 drug resistance gene subtypes have no significant correlation between other indicator bacteria. 
It is worth noting that potential hosts of oqxB, mexD and sul2, including Thauera, Pseudomonas and Acinetobacter, were identified as major contributors to mobile ARGs in the samples above. Additionally, we analyzed the association between the overall indicator bacteria and AACs, and the results showed that sul2 was the most strongly associated mobile drug resistance gene with the indicator bacteria (Fig. S5).

\section{Discussion}

\section{The shifts of bacterial communities in different types of water bodies}

Our study revealed that there were significant differences in microbial diversity in water bodies subject to different degrees of anthropogenic interference. The microbial diversity of group $D$ was the lowest of all groups, which may be attributed to the reception of a large amount of domestic sewage and aquaculture wastewater, indicating that a significant decrease in microbial diversity may occur in anthropogenic interference areas. The possible reason is that the increase in the load of environmental biological and organic pollutants under the condition of fecal pollution, which poses a specific selection pressure on the microbial community, thus leads to the decrease of microbial diversity (Liu et al. 2018). Proteobacteria was the most abundant phylum in all samples, and its abundance in the wastewater group is significantly higher than that in the river group. Several phyla such as Cyanobacteria and Actinobacteria showed significant enrichment in river water, similar results were observed in recent study on Chaohu Lake (Zhang et al. 2020). The authors found that the abundance of Cyanobacteria and Actinobacteria was significantly correlated with total nitrogen (TN) and total phosphorus (TP), suggest that the river selected by this study has been affected by eutrophication-related problems. By contrast, the sewage group also observed the enrichment of Firmicutes, these microorganisms are often detected in the wastewater treatment system (de Celis et al. 2020), and some of its members have been proved to play an important role in specific ecosystem functions such as nutrient removal (Johnson et al. 2015).

The distribution pattern of potential pathogens in water samples was characterized through the local $16 \mathrm{~S}$ rDNA database of potential pathogens. All the detected potential pathogens were clustered into 34 genera, and the most abundant sequences of potential pathogens were assigned to Bartonella, accounting for $25.23 \%$ of the total potential pathogen abundance. As expected, site $D$ harboring the highest level of potential pathogens, which could explained by the large amount of domestic wastewater received by this location. In addition, some potential pathogens such as Aeromonas and Comamonas did not show a decrease in relative abundance after sewage treatment, while Pseudomonas and Mycobacterium found an increase in relative abundance downstream of the sewage outlet. In particular, Bacillus was detected in the WWTP effluent and ecological interception ditch rather than WWTP influent. These species may survive in the sewage treatment system and contribute to sewage treatment. For example, Pseudomonas resists environmental stress by producing extracellular polymeric substances (EPS), including polysaccharides, extracellular DNAs (eDNA), and proteins (Kim and Lee 2016), Aeromonas can coordinate biofilm formation through QS systems (Talagrand-Reboul et al. 2017), and Comamonas, as one of the most abundant members of the biofilm community in wastewater treatment facilities, plays an important role in the degradation of various organic compounds (Wu et al. 2015), 
Bacillus is also a member of biofilm bacteria in the wastewater system, which is essential for the formation of efficient bioaugmentation systems (Cheng et al. 2015). The biofilm process plays an important role in wastewater treatment (Rathnaweera et al. 2020), but after long-term development, the biofilm may become reservoirs for pathogens and ARGs. Furthermore, high relative abundance of Enterobacter was found in all sites of the FR group, and Klebsiella was also detected in four of these sites, both of which contain classical intestinal pathogens (Cui et al. 2019), indicating that FR may be contaminated by faeces. These potential pathogens may have obtained antibiotic resistance genes in sewage treatment facilities (Munck et al. 2015), and the potential health risks can not be ignored.

\section{Distribution patterns of ARGs and MGEs}

We found that the MDR efflux pump gene was the most abundant ARGs in all samples, which was similar to the previous study (Rodríguez et al. 2021), followed by aminoglycoside, fluoroquinolone and isoniazid resistance genes, in which aminoglycoside is the first antibiotics discovered and used clinically, although its importance has diminished due to the emergence of other broad-spectrum antibiotics, but it is still an effective choice against multidrug-resistant bacteria (Becker and Cooper 2013). Fluoroquinolone is one of the most widely used antibiotics in China in 2013 (Zhang et al. 2015), while isoniazid is not a commonly used type of antibiotics, and the increase in its abundance may be related to the frequency of local use. It is worth noting that polymyxin resistance gene was detected in all samples, which has been regarded as "last resort" antimicrobials because of its important value in the clinical treatment of multidrug resistant bacterial infections (Trimble et al. 2016), indicating that there may be local antibiotic abuse. Some subtypes of glycopeptide resistance gene did not show a decrease in abundance at the WWTP effluent, which may be due to the poor removal rate of some bacteria with strong environmental resistance in the sewage treatment system, and these bacteria may be the hosts of multiple drug resistance genes. Some glycopeptide resistance gene found in this study, such as vanA, vanB, vanC, vanL, are usually found in enterococci (Oravcova et al. 2017), and such bacteria should be included in the scope of monitoring in the follow-up study.

In order to determine the potential of horizontal transfer of ARGs between bacteria, we evaluated the abundance of movable elements including prophages, plasmids and insertion sequences in all samples. D had the highest relative abundance of MGEs, followed by FR. In particular, the level of MGEs decreased significantly after sewage treatment. Previous studies revealed that the anaerobic digestion process has the potential to reduce insertion sequences and plasmids (Miller et al. 2016), which may be the reason for the significant decrease in MGEs levels at the WWTP effulent in the study. MGEs, which ubiquitous in prokaryotes, plays an important role in the transmission of ARGs and virulence factors in bacterial communities through horizontal gene transfer (Piotrowska and Popowska 2015). The coexistence of MGEs and ARGs will increase the possibility of ARGs transfer from drug-resistant bacteria to pathogens, thus increasing health risks.

\section{Host identification of ARGs}


In order to determining the taxonomic origin of ARGs, we identified the host of AACs. 54 of ARG subtypes were assigned to Escherichia coli, which has a strong ability to accumulate resistance genes, mostly through HGT, MDR Escherichia coli could lead to difficult-totreat infections (Poirel et al. 2018). 39 ARG subtypes were assigned to Bacteroides graminisolvens, which was originally isolated from the anaerobic sludge of cattle farm waste, cells of the strain were gram-negative, non-motile, non-spore-forming rods (Nishiyama et al. 2009). In our study, these ARG-carrying species was mainly distributed in wastewater groups. In particular, it was found that 20 potential pathogenic species carrying ARGs, 36 ARG subtypes were assigned to Arcobacter butzleri and Stenotrophomonas maltophilia, where Arcobacter butzleri, an emerging food-borne and water-borne zoonotic pathogen that can cause human diarrhea (Kietsiri et al. 2021), Stenotrophomonas maltophilia is the most commonly identified bacterial microorganism in respiratory tract cultures (Chong et al. 2021), while 17 ARG subtypes are assigned to Pseudomonas alcaligenes, a common opportunistic pathogen (Valenstein et al. 1983). The horizontal gene transfer between indigenous environmental bacteria and the selective pressure caused by antibiotics and heavy metals in wastewater may be the inducement for the existence of ARGs in potential pathogens (Guo et al. 2017). Additionally, ARGs and MGEs carrying Contigs were mainly assigned to Thauera, Pseudomonas, Escherichia and Acinetobacter. Thauera is the dominant genus in activated sludge, as an aromaticdegrading bacteria (Miran et al. 2018), it has been proved to have strong adaptability to antibiotic pressure in previous studies (Zhao et al. 2019). The main subtype of mobile ARGs carried by Thauera was Escherichia coli EF-Tu mutants conferring resistance to Enacyloxin lia, accounting for $98 \%$ of the total abundance of mobile ARGs. EF-Tu was one of the most abundant proteins in bacterial cells (Prezioso et al. 2017), and its mutation gives bacteria resistance to elfamycin.

It is worth noting that of the 113 genera annotated to carrying highly mobile ARGs, 25 carried Escherichia coli EF-Tu mutants conferring resistance to Enacyloxin lia. Recent study indicate that, the EF-Tu mutant was the most abundant ARG in WWTPs effluents (Bondarczuk and Piotrowska-Seget 2019), which was similar to this study. Several species of Acinetobacter can cause infection in immunocompromised human hosts, and some of these members, such as Acinetobacter baumannii, have been shown to have the potential to develop multidrug resistance (Lee et al. 2017). Pseudomonas contains a variety of inherent and acquired ARGs and has the ability to exchange ARGs with some gram-negative-bacilli such as Enterobacteriacea (Kittinger et al. 2016), some members of this genus, such as Pseudomonas Aeruginosa, Pseudomonas putida and Pseudomonas fluorescens, were clinically important opportunistic pathogens (Silby et al. 2011). In particular, Pseudomonas, carbapenem-resistant Enterobacteriaceae, and Acinetobacterwere listed by World Health Organization (WHO) as the bacteria most urgently in need of new antibiotics (http://www.who.int/medicines/publications/global-priority-list-antibiotic-resistantbacteria/en/).

\section{Association between ARGs, potential pathogens and indicator bacteria}

WWTP influent had the highest relative abundance of pollution indicator microorganisms, and similarly, this site also harbored the highest level of potential pathogens and ARGs. This site receives a large amount of domestic sewage and agricultural wastes, human and other environmental sources of bacteria 
gather in the wastewater, thus improving the level of pathogenicity. Although it could not be completely eliminated, a significant decrease in the level of indicator bacteria was found in sewage outlets and ecological ditches. Pearson correlation analysis indicated that potential pathogens were highly associated with FIB, but not with TC, which echoes previous sentiments that FIB is recommended as an alternative indicator of microbial risk in water environment (Yergeau et al. 2016). Network analysis provides a new perspective for studying the distribution and occurrence of environmental drug resistance genes (Li et al. 2015).

Our study revealed that FIB and TC were significantly associated with 10 and 20 drug resistance genes, respectively, while dfrE, sul2, mexD, PmrE, oprA were closely associated with indicator bacteria, in which mexD and oprA belong to MRD efflux pump were often detected in Pseudomonas aeruginosa (Zahedi bialvaei et al. 2021). Interestingly, we found that there was a close relationship between PmrE and indicator taxa, which may be related to the frequent detection of polymyxin resistance genes in all samples. Sul2 endowed with resistance to fluoroquinolones has been widely reported in fresh water. As an important indicator of fresh water pollution, it may be derived from microorganisms that degrade sulfonamides (Nnadozie and Odume 2019). It is reported that most sulfa-degrading bacteria may be resistant to sulfonamides and may carry sulfonamide resistance genes including sul1 and sul2 (VilaCosta et al. 2017). Many studies have focused on Escherichia coli because it is considered to have both the ability to indicate fecal contamination and the ability to assess the spread of antibiotic resistance in water environment (Triggiano et al. 2020). Such indicator microorganisms, especially the coexistence of coliform and potential pathogens, may promote the transfer of ARGs to potential pathogens and further increase the risk of MDR pathogens (Mishra et al. 2018). Obviously more studies should better reveal the mechanism of ARGs acquired by potential pathogens in the environment.

\section{Conclusion}

In this study, we characterized the composition of environmental species, including indicator microorganisms and potential pathogens, and further performed a metagenomic analysis using a whole metagenome shotgun approach, to explore the distribution and potential host of ARGs. In the anthropogenically impacted environment, the microbial diversity decreased significantly, and showed the enrichment of specific bacteria, such as Proteobacteria and Firmicutes, etc. Different levels of potential pathogens were detected in all sampling sites, some potential pathogens with strong environmental pressure resistance, including Aeromona, Comamonas, Pseudomonas and Mycobacterium, could not be well eliminated by sewage treatment, in which Pseudomonas was annotated as a potential hot spot of horizontal transfer of antibiotic resistance genes in the environment. The survival and spread of such bacteria in the environment may bring increased public health hazards. In addition, we also found that although there was a significant correlation between microbial indicators (FIB and TC) and potential pathogens, the correlation of FIB was more significant, which emphasized the advantage of FIB as a public health risk index of water body. For potential pathogens, TC showed only a relatively weak association, but some of its members, such as Escherichia coli and Klebsiella, showed association with a variety of ARGs, so the monitoring of TC is still of great significance. Overall, it is suggested that the 
assessment of antibiotic resistance indicating bacteria should be incorporated into the monitoring framework of environmental microbial risk to better characterize the risk of environmental pathogenicity.

\section{Declarations}

\section{Funding}

This study was supported by the University Synergy Innovation Program of Anhui Province (Grant No. GXXT-2019-035) and Integration and Demonstration of Quality and Safety Control Technology for Green Ecological Livestock and Poultry Products Industry Chain (1604a0702033).

\section{Conflict of interest}

The authors declare no conflict of interest.

\section{Ethics approval and consent to participate}

This study was performed in accordance with the Chinese Laboratory Animal Administration Act of 1988. Before experiments, the research protocol was reviewed and approved by the Research Ethics Committee of Anhui Agricultural University. Permission was obtained from all managers on study farms before sampling.

\section{Consent for publication}

Not applicable.

\section{Data Avaliable}

The original sequencing data has been uploaded to NCBI Sequence Read Archive (SRA), study accession number: PRJNA672794.

\section{Authors' contributions}

Ke-Zong Qi and Yin Shao conceived the idea. Zhao Qi, Yin Shao and Xiang-Jun Song collected the samples. Xiang-Long Zhao, Hao Huang and Jian Tu analyzed and interpreted the data. Xiang-Long Zhao wrote the manuscript. All authors read and approved the final manuscript.

\section{Acknowledgements}

We thank the University Synergy Innovation Program of Anhui Province for supporting the highthroughput sequencing. The collection of the experimental samples was supported by Integration and Demonstration of Quality and Safety Control Technology for Green Ecological Livestock and Poultry Products Industry Chain.

\section{Authors' information}


Anhui Province Key Laboratory of Veterinary Pathobiology and Disease Control, Anhui Agricultural University, Hefei, 230036, P. R. China

Xiang-Long Zhao, Zhao Qi, Hao Huang, Jian Tu, Xiang-Jun Song, Ke-Zong Qi, Yin Shao

Contributions

Both authors made a direct intellectual contribution and approved the publication of the manuscript.

Corresponding authors

Correspondence to Ke-Zong Qi and Yin Shao

\section{References}

1. Ahmed J, Wong LP, Chua YP, et al (2020) Quantitative Microbial Risk Assessment of Drinking Water Quality to Predict the Risk of Waterborne Diseases in Primary-School Children. IJERPH 17:2774. https://doi.org/10.3390/ijerph17082774

2. Altschul S (1997) Gapped BLAST and PSI-BLAST: a new generation of protein database search programs. Nucleic Acids Research 25:3389-3402. https://doi.org/10.1093/nar/25.17.3389

3. Ahmed W, Harwood VJ, Nguyen K, et al (2016) Utility of Helicobacter spp. associated GFD markers for detecting avian fecal pollution in natural waters of two continents. Water Research 88:613-622. https://doi.org/10.1016/j.watres.2015.10.050

4. Byappanahalli MN, Nevers MB, Korajkic A, et al (2012) Enterococci in the Environment. Microbiol Mol Biol Rev 76:685-706. https://doi.org/10.1128/MMBR.00023-12

5. Bush K, Courvalin P, Dantas G, et al (2011) Tackling antibiotic resistance. Nat Rev Microbiol 9:894896. https://doi.org/10.1038/nrmicro2693

6. Becker B, Cooper MA (2013) Aminoglycoside Antibiotics in the 21st Century. ACS Chem Biol 8:105115. https://doi.org/10.1021/cb3005116

7. Bondarczuk K, Piotrowska-Seget Z (2019) Microbial diversity and antibiotic resistance in a final effluent-receiving lake. Science of The Total Environment 650:2951-2961. https://doi.org/10.1016/j.scitotenv.2018.10.050

8. Chen $\mathrm{H}$, Chen $\mathrm{R}$, Jing $\mathrm{L}$, et al (2019) A metagenomic analysis framework for characterization of antibiotic resistomes in river environment: Application to an urban river in Beijing. Environmental Pollution 245:398-407. https://doi.org/10.1016/j.envpol.2018.11.024

9. Cui Q, Huang $Y$, Wang $H$, Fang $T$ (2019) Diversity and abundance of bacterial pathogens in urban rivers impacted by domestic sewage. Environmental Pollution 249:24-35. https://doi.org/10.1016/j.envpol.2019.02.094

10. Cheng $Z$, Chen $M$, Xie $L$, et al (2015) Bioaugmentation of a sequencing batch biofilm reactor with Comamonas testosteroni and Bacillus cereus and their impact on reactor bacterial communities. 
Biotechnol Lett 37:367-373. https://doi.org/10.1007/s10529-014-1684-1

11. Chong WH, Saha BK, Ananthakrishnan Ramani, Chopra A (2021) State-of-the-art review of secondary pulmonary infections in patients with COVID-19 pneumonia. Infection.

https://doi.org/10.1007/s15010-021-01602-z

12. Cai L, Zhang T (2013) Detecting Human Bacterial Pathogens in Wastewater Treatment Plants by a High-Throughput Shotgun Sequencing Technique. Environ Sci Technol 47:5433-5441. https://doi.org/10.1021/es400275r

13. Duan C, Cui Y, Zhao Y, et al (2016) Evaluation of Faecalibacterium 16S rDNA genetic markers for accurate identification of swine faecal waste by quantitative PCR. Journal of Environmental Management 181:193-200. https://doi.org/10.1016/j.jenvman.2016.06.022

14. Devane ML, Moriarty E, Weaver L, et al (2020) Fecal indicator bacteria from environmental sources; strategies for identification to improve water quality monitoring. Water Research 185:116204. https://doi.org/10.1016/j.watres.2020.116204

15. Wang D, Li HF, Liu F, Wang Y, Zhong YC, He Y, Xiao RF, Wu JS (2016) Interception Effect of Ecological Ditch on Nitrogen Transport in Agricultural Runoff in Subtropical China. Huan Jing Ke Xue. 15;37(5):1717-23 (in Chinese). PMID: 27506024.

16. de Celis M, Belda I, Ortiz-Álvarez R, et al (2020) Tuning up microbiome analysis to monitor WWTPs' biological reactors functioning. Sci Rep 10:4079. https://doi.org/10.1038/s41598-020-61092-1

17. Figueras MJ, Borrego JJ (2010) New Perspectives in Monitoring Drinking Water Microbial Quality. IJERPH 7:4179-4202. https://doi.org/10.3390/ijerph7124179

18. Fang T, Cui Q, Huang Y, et al (2018) Distribution comparison and risk assessment of free-floating and particle-attached bacterial pathogens in urban recreational water: Implications for water quality management. Science of The Total Environment 613-614:428-438.

https://doi.org/10.1016/j.scitotenv.2017.09.008

19. Fu L, Niu B, Zhu Z, et al (2012) CD-HIT: accelerated for clustering the next-generation sequencing data. Bioinformatics 28:3150-3152. https://doi.org/10.1093/bioinformatics/bts565

20. Guo J, Li J, Chen H, et al (2017) Metagenomic analysis reveals wastewater treatment plants as hotspots of antibiotic resistance genes and mobile genetic elements. Water Research 123:468-478. https://doi.org/10.1016/j.watres.2017.07.002

21. Harwood VJ, Staley C, Badgley BD, et al (2014) Microbial source tracking markers for detection of fecal contamination in environmental waters: relationships between pathogens and human health outcomes. FEMS Microbiol Rev 38:1-40. https://doi.org/10.1111/1574-6976.12031

22. Jensen LJ, Julien P, Kuhn M, et al (2007) eggNOG: automated construction and annotation of orthologous groups of genes. Nucleic Acids Research 36:D250-D254. https://doi.org/10.1093/nar/gkm796

23. Johnson DR, Helbling DE, Lee TK, et al (2015) Association of Biodiversity with the Rates of Micropollutant Biotransformations among Full-Scale Wastewater Treatment Plant Communities. Appl Environ Microbiol 81:666-675. https://doi.org/10.1128/AEM.03286-14 
24. Jia B, Raphenya AR, Alcock B, et al (2017) CARD 2017: expansion and model-centric curation of the comprehensive antibiotic resistance database. Nucleic Acids Res 45:D566-D573. https://doi.org/10.1093/nar/gkw1004

25. Korajkic A, McMinn B, Harwood V (2018) Relationships between Microbial Indicators and Pathogens in Recreational Water Settings. IJERPH 15:2842. https://doi.org/10.3390/ijerph15122842

26. Kietsiri P, Muangnapoh C, Lurchachaiwong W, et al (2021) Characterization of Arcobacter spp. Isolated from human diarrheal, non-diarrheal and food samples in Thailand. PLoS ONE 16:e0246598. https://doi.org/10.1371/journal.pone.0246598

27. Kittinger C, Lipp M, Baumert R, et al (2016) Antibiotic Resistance Patterns of Pseudomonas spp. Isolated from the River Danube. Front Microbiol 7:. https://doi.org/10.3389/fmicb.2016.00586

28. Lu X, Zhang X-X, Wang Z, et al (2015) Bacterial Pathogens and Community Composition in Advanced Sewage Treatment Systems Revealed by Metagenomics Analysis Based on HighThroughput Sequencing. PLoS ONE 10:e0125549. https://doi.org/10.1371/journal.pone.0125549

29. Li J, Chen Q, Li H, et al (2020) Impacts of different sources of animal manures on dissemination of human pathogenic bacteria in agricultural soils. Environmental Pollution 266:115399. https://doi.org/10.1016/j.envpol.2020.115399

30. Li R, Li Y, Kristiansen K, Wang J (2008) SOAP: short oligonucleotide alignment program. Bioinformatics 24:713-714. https://doi.org/10.1093/bioinformatics/btn025

31. Liu S, Wang C, Wang P, et al (2018) Variation of bacterioplankton community along an urban river impacted by touristic city: With a focus on pathogen. Ecotoxicology and Environmental Safety 165:573-581. https://doi.org/10.1016/j.ecoenv.2018.09.006

32. Lee C-R, Lee JH, Park M, et al (2017) Biology of Acinetobacter baumannii: Pathogenesis, Antibiotic Resistance Mechanisms, and Prospective Treatment Options. Front Cell Infect Microbiol 7:. https://doi.org/10.3389/fcimb.2017.00055

33. Li B, Yang Y, Ma L, et al (2015) Metagenomic and network analysis reveal wide distribution and cooccurrence of environmental antibiotic resistance genes. ISME J 9:2490-2502. https://doi.org/10.1038/ismej.2015.59

34. Mishra M, Arukha AP, Patel AK, et al (2018) Multi-Drug Resistant Coliform: Water Sanitary Standards and Health Hazards. Front Pharmacol 9:311. https://doi.org/10.3389/fphar.2018.00311

35. Munck C, Albertsen M, Telke A, et al (2015) Limited dissemination of the wastewater treatment plant core resistome. Nat Commun 6:8452. https://doi.org/10.1038/ncomms9452

36. Miller JH, Novak JT, Knocke WR, Pruden A (2016) Survival of Antibiotic Resistant Bacteria and Horizontal Gene Transfer Control Antibiotic Resistance Gene Content in Anaerobic Digesters. Front Microbiol 7:. https://doi.org/10.3389/fmicb.2016.00263

37. Miran W, Jang J, Nawaz M, et al (2018) Biodegradation of the sulfonamide antibiotic sulfamethoxazole by sulfamethoxazole acclimatized cultures in microbial fuel cells. Science of The Total Environment 627:1058-1065. https://doi.org/10.1016/j.scitotenv.2018.01.326 
38. Neher TP, Ma L, Moorman TB, et al (2020) Seasonal variations in export of antibiotic resistance genes and bacteria in runoff from an agricultural watershed in lowa. Science of The Total Environment 738:140224. https://doi.org/10.1016/j.scitotenv.2020.140224

39. Noguchi $H$, Park J, Takagi $T$ (2006) MetaGene: prokaryotic gene finding from environmental genome shotgun sequences. Nucleic Acids Research 34:5623-5630. https://doi.org/10.1093/nar/gkl723

40. Nishiyama T, Ueki A, Kaku N, et al (2009) Bacteroides graminisolvens sp. nov., a xylanolytic anaerobe isolated from a methanogenic reactor treating cattle waste. INTERNATIONAL JOURNAL OF SYSTEMATIC AND EVOLUTIONARY MICROBIOLOGY 59:1901-1907. https://doi.org/10.1099/ijs.0.008268-0

41. Nnadozie CF, Odume ON (2019) Freshwater environments as reservoirs of antibiotic resistant bacteria and their role in the dissemination of antibiotic resistance genes. Environmental Pollution 254:113067. https://doi.org/10.1016/j.envpol.2019.113067

42. Oravcova V, Mihalcin M, Zakova J, et al (2017) Vancomycin-resistant enterococci with vanA gene in treated municipal wastewater and their association with human hospital strains. Science of The Total Environment 609:633-643. https://doi.org/10.1016/j.scitotenv.2017.07.121

43. Purnell S, Halliday A, Newman F, et al (2020) Pathogen infection risk to recreational water users, associated with surface waters impacted by de facto and indirect potable reuse activities. Science of The Total Environment 722:137799. https://doi.org/10.1016/j.scitotenv.2020.137799

44. Poirel L, Madec J-Y, Lupo A, et al (2018) Antimicrobial Resistance in Escherichia coli. In: Schwarz, Cavaco, Shen (eds) Antimicrobial Resistance in Bacteria from Livestock and Companion Animals. American Society of Microbiology, pp 289-316

45. Piotrowska M, Popowska M (2015) Insight into the mobilome of Aeromonas strains. Front Microbiol 6:. https://doi.org/10.3389/fmicb.2015.00494

46. Prezioso SM, Brown NE, Goldberg JB (2017) Elfamycins: inhibitors of elongation factor-Tu: Elfamycin antibiotics. Molecular Microbiology 106:22-34. https://doi.org/10.1111/mmi.13750

47. Rathnaweera SS, Rusten B, Manamperuma LD, et al (2020) Innovative, compact and energy-efficient biofilm process for nutrient removal from wastewater. Water Science and Technology 81:19411950. https://doi.org/10.2166/wst.2020.245

48. Rodríguez EA, Ramirez D, Balcázar JL, Jiménez JN (2021) Metagenomic analysis of urban wastewater resistome and mobilome: A support for antimicrobial resistance surveillance in an endemic country. Environmental Pollution 276:116736.

https://doi.org/10.1016/j.envpol.2021.116736

49. Schmeller DS, Loyau A, Bao K, et al (2018) People, pollution and pathogens - Global change impacts in mountain freshwater ecosystems. Science of The Total Environment 622-623:756-763. https://doi.org/10.1016/j.scitotenv.2017.12.006

50. Shin SB, Lee JH, Lim CW, et al (2019) Fecal source tracking based on fecal coliform concentration and bacterial community structure in the Bong stream, Korea. Environ Sci Pollut Res 26:5601-5612. https://doi.org/10.1007/s11356-018-3995-6 
51. Silby MW, Winstanley C, Godfrey SAC, et al (2011) Pseudomonas genomes: diverse and adaptable. FEMS Microbiol Rev 35:652-680. https://doi.org/10.1111/j.1574-6976.2011.00269.x

52. The European Union summary report on trends and sources of zoonoses, zoonotic agents and foodborne outbreaks in 2016. EFSA Journal 228

53. Triggiano F, Calia C, Diella G, et al (2020) The Role of Urban Wastewater in the Environmental Transmission of Antimicrobial Resistance: The Current Situation in Italy (2010-2019). Microorganisms 8:1567. https://doi.org/10.3390/microorganisms8101567

54. Tatusov RL, Fedorova ND, Jackson JD, et al (2003) The COG database: an updated version includes eukaryotes. BMC Bioinformatics 14

55. Talagrand-Reboul E, Jumas-Bilak E, Lamy B (2017) The Social Life of Aeromonas through Biofilm and Quorum Sensing Systems. Front Microbiol 8:. https://doi.org/10.3389/fmicb.2017.00037

56. Trimble MJ, Mlynárčik P, Kolář M, Hancock REW (2016) Polymyxin: Alternative Mechanisms of Action and Resistance. Cold Spring Harb Perspect Med 6:a025288.

https://doi.org/10.1101/cshperspect.a025288

57. Valenstein P, Bardy GH, Cox CC, Zwadyk P (1983) Pseudomonas Alcaligenes Endocarditis. American Journal of Clinical Pathology 79:245-247. https://doi.org/10.1093/ajcp/79.2.245

58. Vila-Costa M, Gioia R, Aceña J, et al (2017) Degradation of sulfonamides as a microbial resistance mechanism. Water Research 115:309-317. https://doi.org/10.1016/j.watres.2017.03.007

59. Wang D, Li HF, Liu F, Wang Y, Zhong YC, He Y, Xiao RF, Wu JS. [Interception Effect of Ecological Ditch on Nitrogen Transport in Agricultural Runoff in Subtropical China]. Huan Jing Ke Xue. 2016 May 15;37(5):1717-23. Chinese. PMID: 27506024.

60. Wattam AR, Abraham D, Dalay O, et al (2014) PATRIC, the bacterial bioinformatics database and analysis resource. Nucl Acids Res 42:D581-D591. https://doi.org/10.1093/nar/gkt1099

61. Wu Y, Shukal S, Mukherjee M, Cao B (2015) Involvement in Denitrification is Beneficial to the Biofilm Lifestyle of Comamonas testosteroni : A Mechanistic Study and Its Environmental Implications. Environ Sci Technol 49:11551-11559. https://doi.org/10.1021/acs.est.5b03381

62. World Health Organization (ed) (2011) Guidelines for drinking-water quality, 4th ed. World Health Organization, Geneva

63. Xie C, Mao X, Huang J, et al (2011) KOBAS 2.0: a web server for annotation and identification of enriched pathways and diseases. Nucleic Acids Research 39:W316-W322. https://doi.org/10.1093/nar/gkr483

64. Yue Y, Huang H, Qi Z, et al (2020) Evaluating metagenomics tools for genome binning with real metagenomic datasets and CAMI datasets. BMC Bioinformatics 21:334. https://doi.org/10.1186/s12859-020-03667-3

65. Yang Y, Li B, Ju F, Zhang T (2013) Exploring Variation of Antibiotic Resistance Genes in Activated Sludge over a Four-Year Period through a Metagenomic Approach. Environ Sci Technol 47:1019710205. https://doi.org/10.1021/es4017365 
66. Yang Z, Kong F, Zhang M (2016) Groundwater contamination by microcystin from toxic cyanobacteria blooms in Lake Chaohu, China. Environ Monit Assess 188:280. https://doi.org/10.1007/s10661-016-5289-0

67. Yang X, Cui H, Liu X, et al (2020) Water pollution characteristics and analysis of Chaohu Lake basin by using different assessment methods. Environ Sci Pollut Res 27:18168-18181. https://doi.org/10.1007/s11356-020-08189-2

68. Yergeau E, Masson L, Elias M, et al (2016) Comparison of Methods to Identify Pathogens and Associated Virulence Functional Genes in Biosolids from Two Different Wastewater Treatment Facilities in Canada. PLoS ONE 11:e0153554. https://doi.org/10.1371/journal.pone.0153554

69. Zhang L, Fang W, Li X, et al (2020) Linking bacterial community shifts with changes in the dissolved organic matter pool in a eutrophic lake. Science of The Total Environment 719:137387. https://doi.org/10.1016/j.scitotenv.2020.137387

70. Zhang Q-Q, Ying G-G, Pan C-G, et al (2015) Comprehensive Evaluation of Antibiotics Emission and Fate in the River Basins of China: Source Analysis, Multimedia Modeling, and Linkage to Bacterial Resistance. Environ Sci Technol 49:6772-6782. https://doi.org/10.1021/acs.est.5b00729

71. Zhao R, Feng J, Liu J, et al (2019) Deciphering of microbial community and antibiotic resistance genes in activated sludge reactors under high selective pressure of different antibiotics. Water Research 151:388-402. https://doi.org/10.1016/j.watres.2018.12.034

72. Zahedi bialvaei A, Rahbar M, Hamidi-Farahani R, et al (2021) oprA-Expression of RND efflux pumps mediated antibiotic resistance in Pseudomonas aeruginosa clinical strains. Microbial Pathogenesis 153:104789. https://doi.org/10.1016/j.micpath.2021.104789

\section{Figures}


a

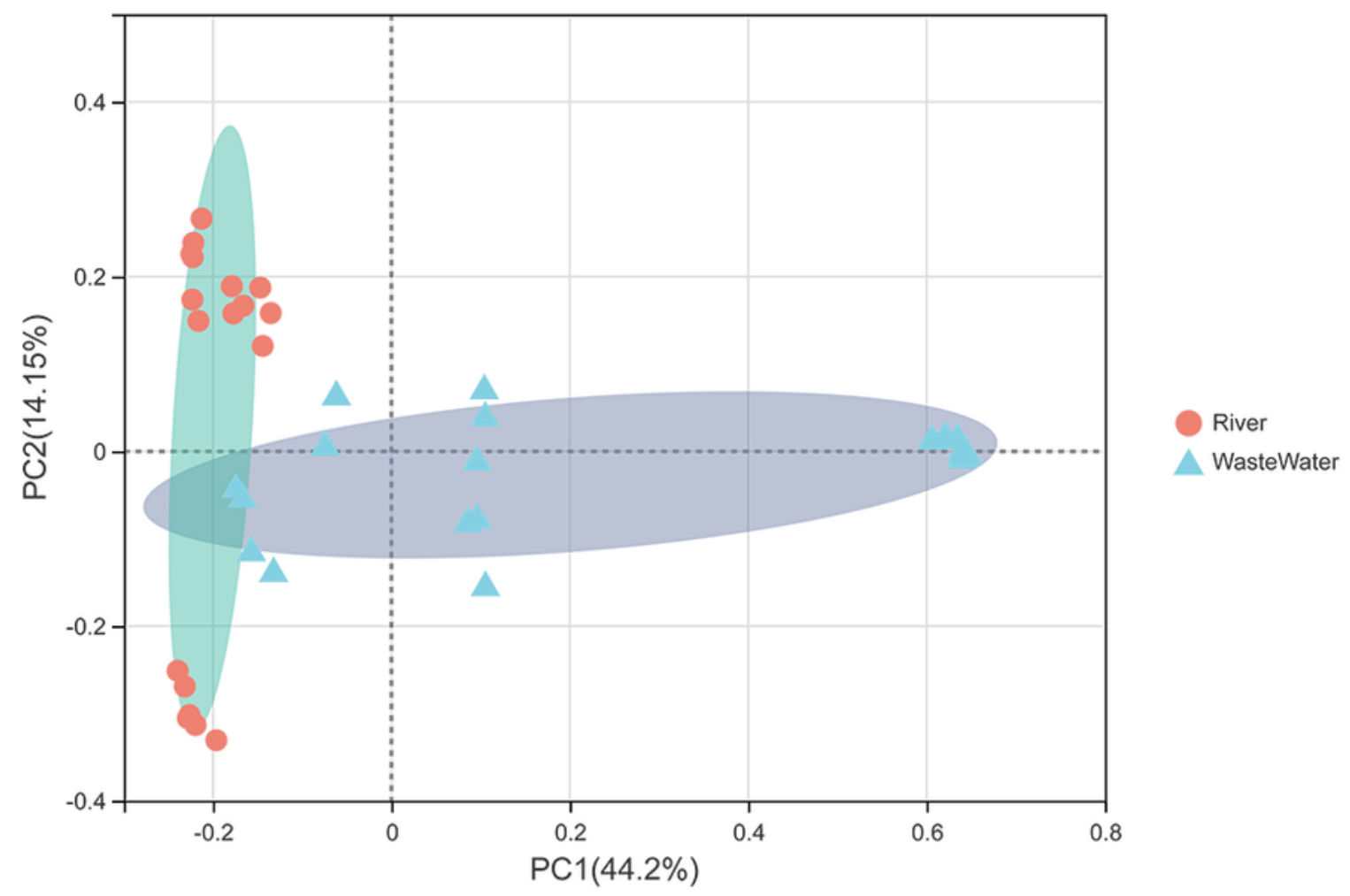

b

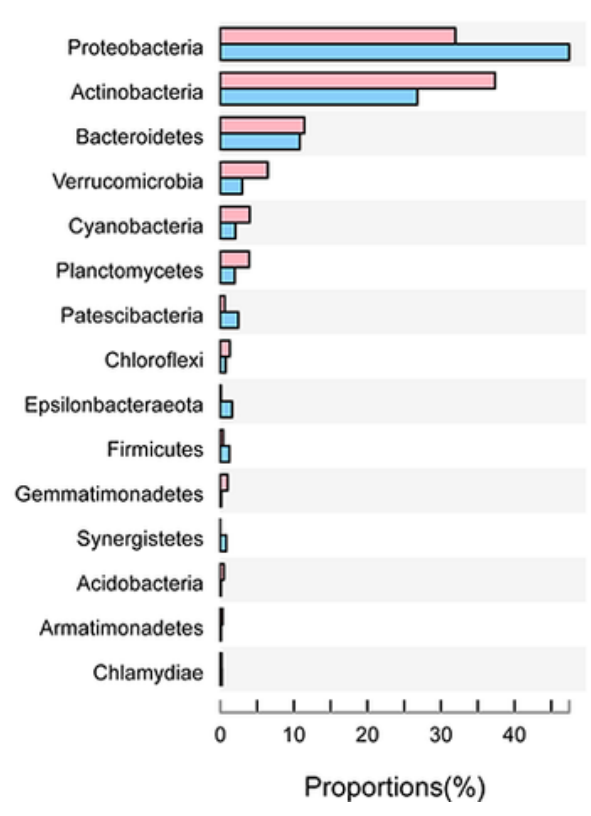

$95 \%$ confidence intervals

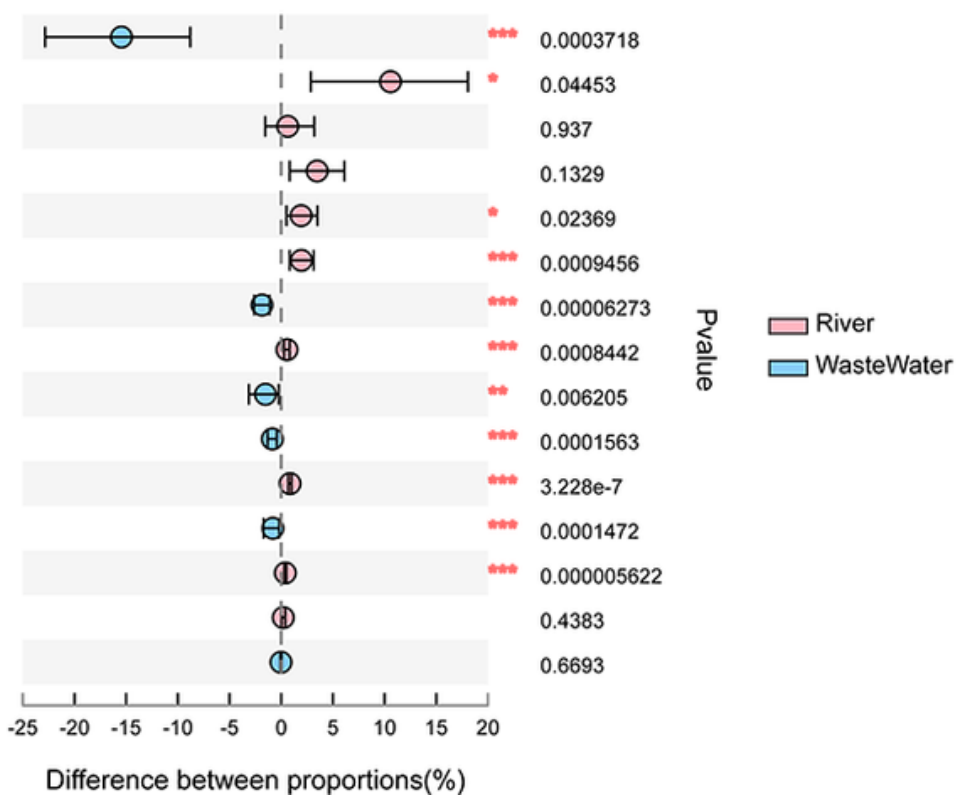

\section{Figure 1}

(a) Principal coordinates analysis (PCoA) of the microbial community in different group. Derived from Bray-Curtis dissimilarity between water samples. (b) Significantly different relative abundances between the WWTP sewage and the River water in phylum level. The one-way ANOVA test was used to evaluate the importance of comparisons between riverwater and wastewater groups. $* \mathrm{P}<0.05, * * \mathrm{P}<0.01$, and $* * * \mathrm{P}<0.001$. 


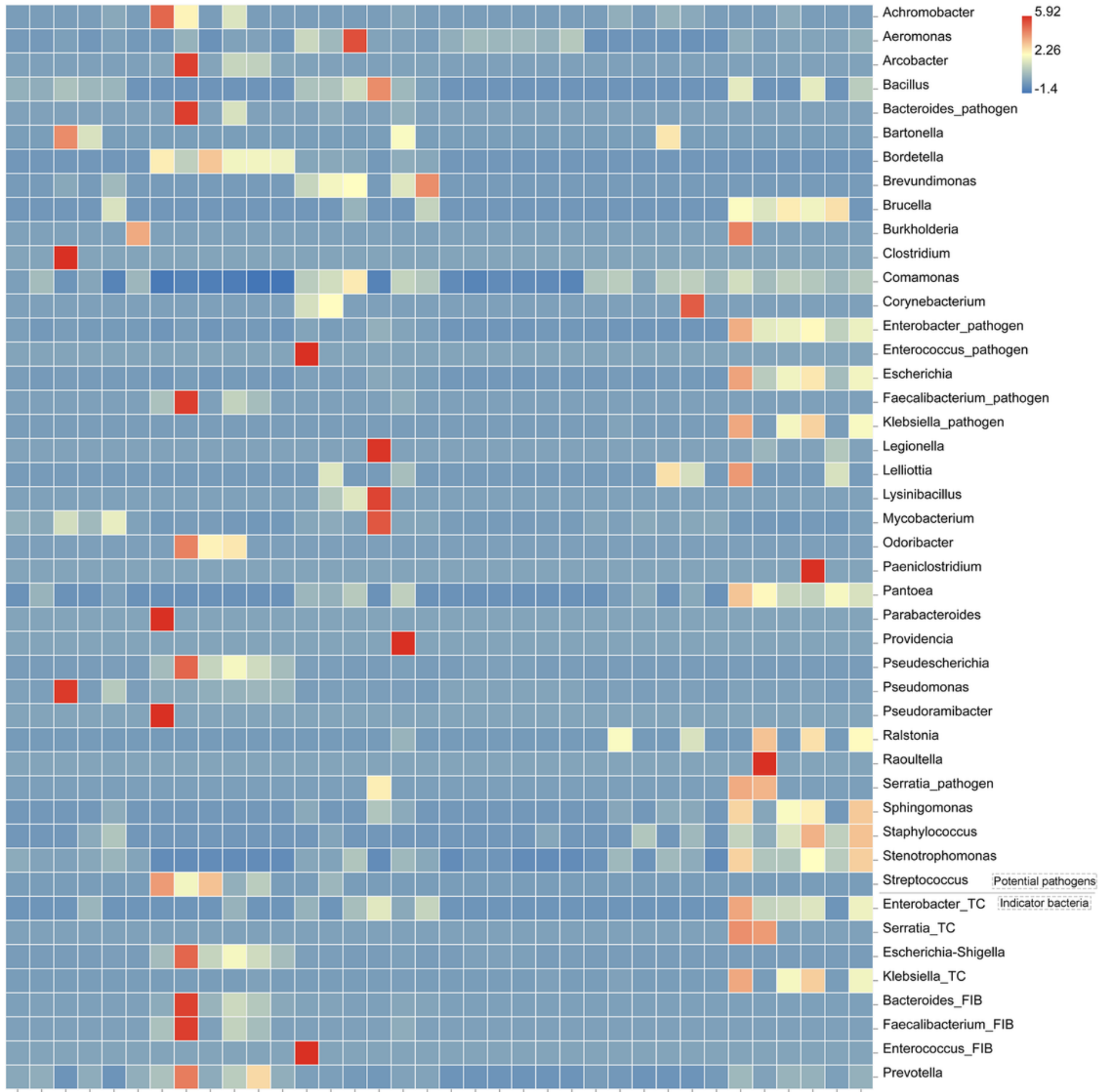

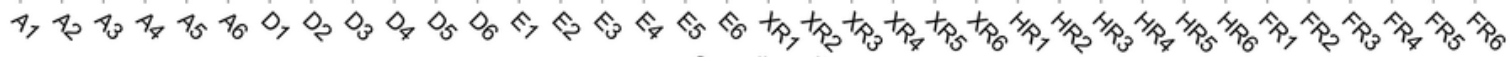
Sampling sites

\section{Figure 2}

Relative abundance of fecal indicator bacteria and potential pathogens observed in all samples. The categories above the gray lines belong to potential pathogens and those under the lines belong to indicator bacteria. 


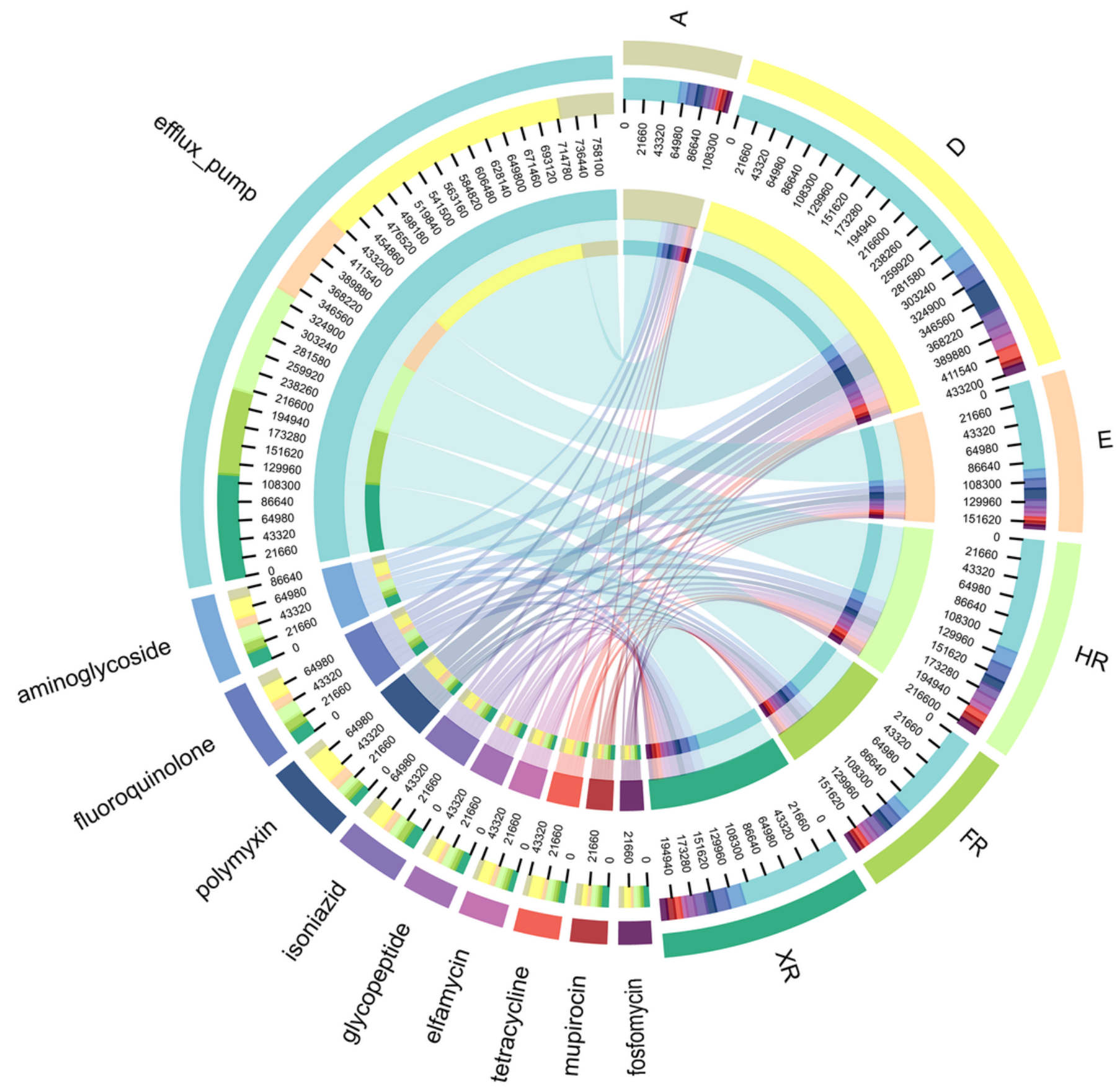

Figure 3

Circos diagram showing the abundance distribution of each ARG type in WWTP influent (D), WWTP effluent (E), Ecological interception ditch (A), Hangbu river (HR), Fengle river (FR) and Paihe river (XR). 


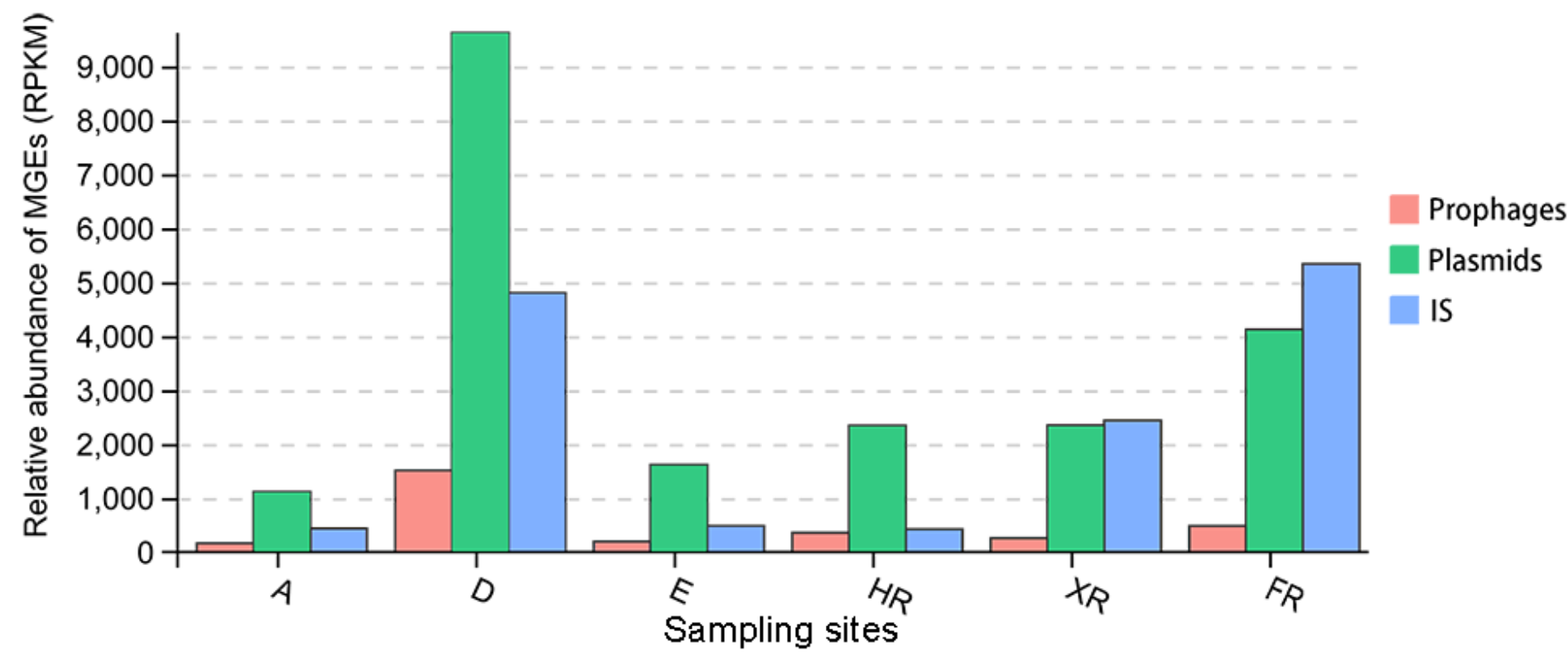

Figure 4

Relative abundance of MGEs in all samples. The reads per kilobase per million mapped reads (RPKM) represented the abundance. 


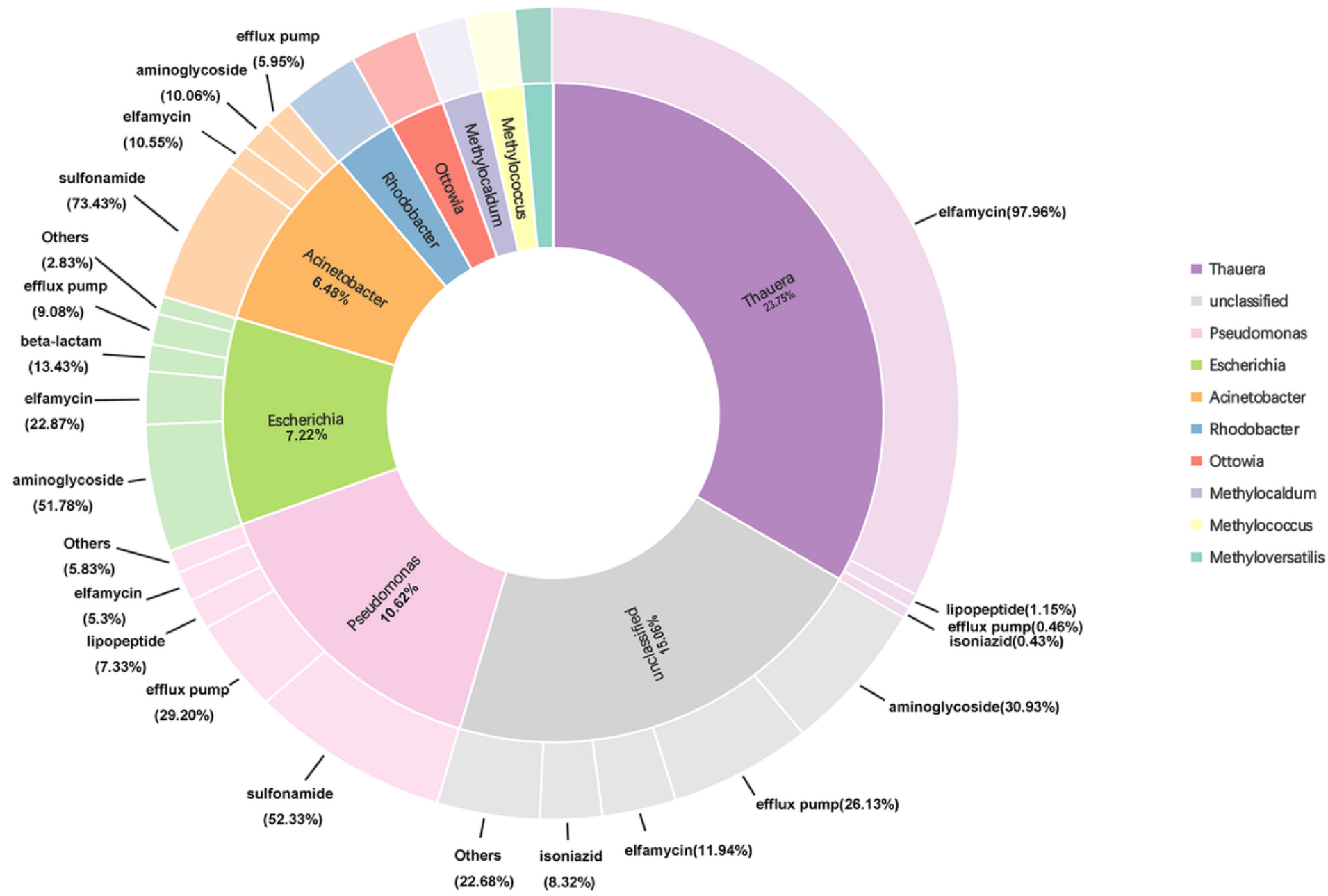

\section{Figure 5}

Taxonomy of ARG- and MGE -carrying contigs at the genus level. The inner circle represents the annotation of the potential mobile ARG host at the genus level. The outer circle represents the composition of the ARG types. 


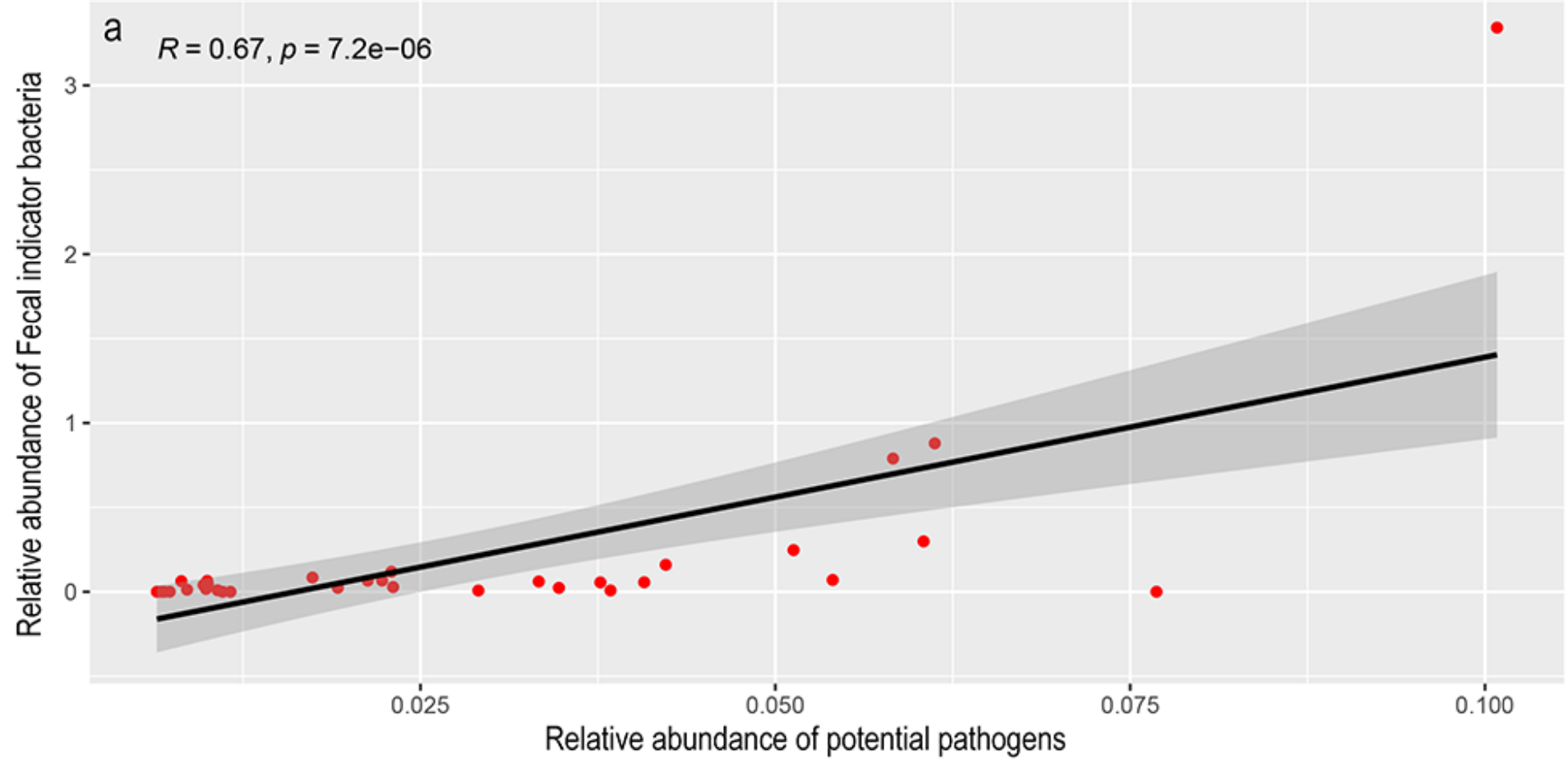

$2.0-\mathrm{b} \quad R=0.49, p=0.0025$

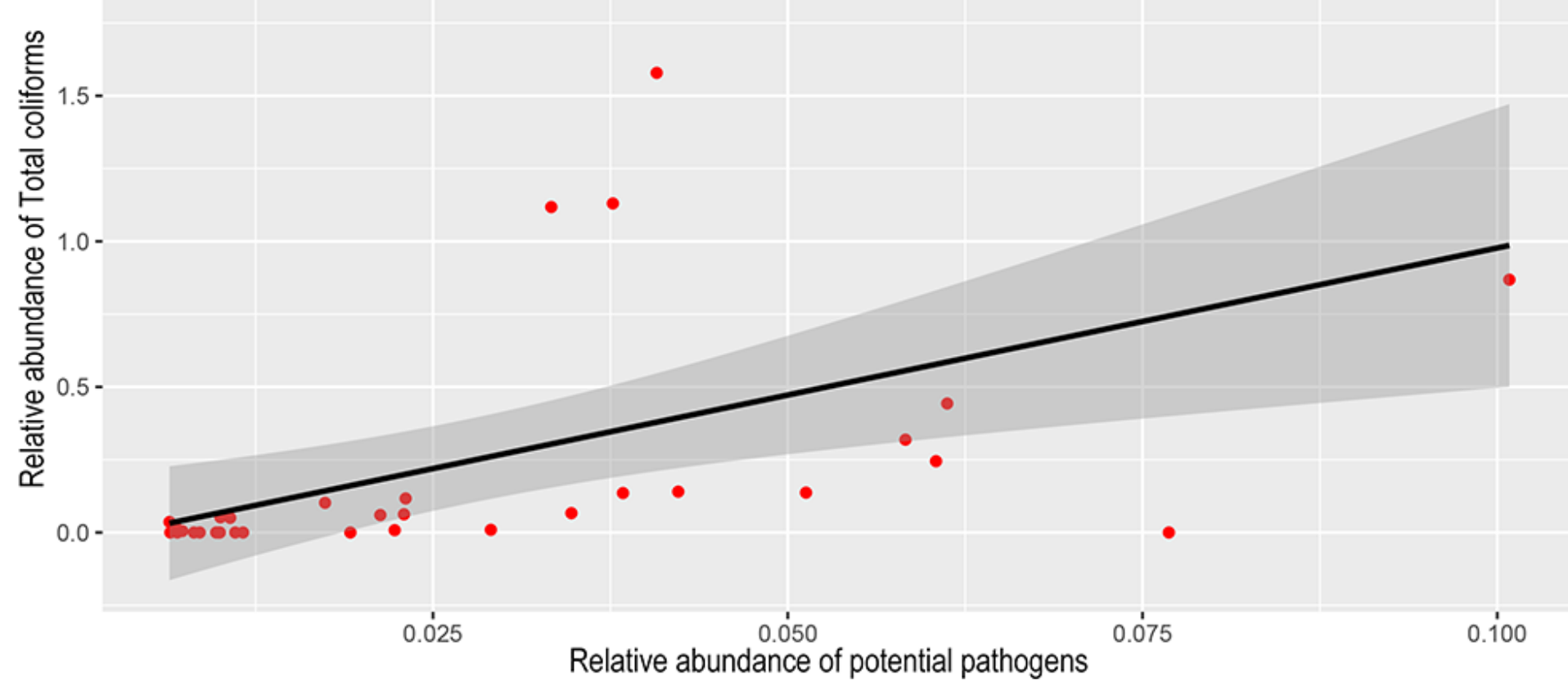

Figure 6

Correlation analysis between indicator bacteria and potential pathogen: (a) The correlation between FIB and potential pathogens. (b) The correlation between TC and potential pathogens. 


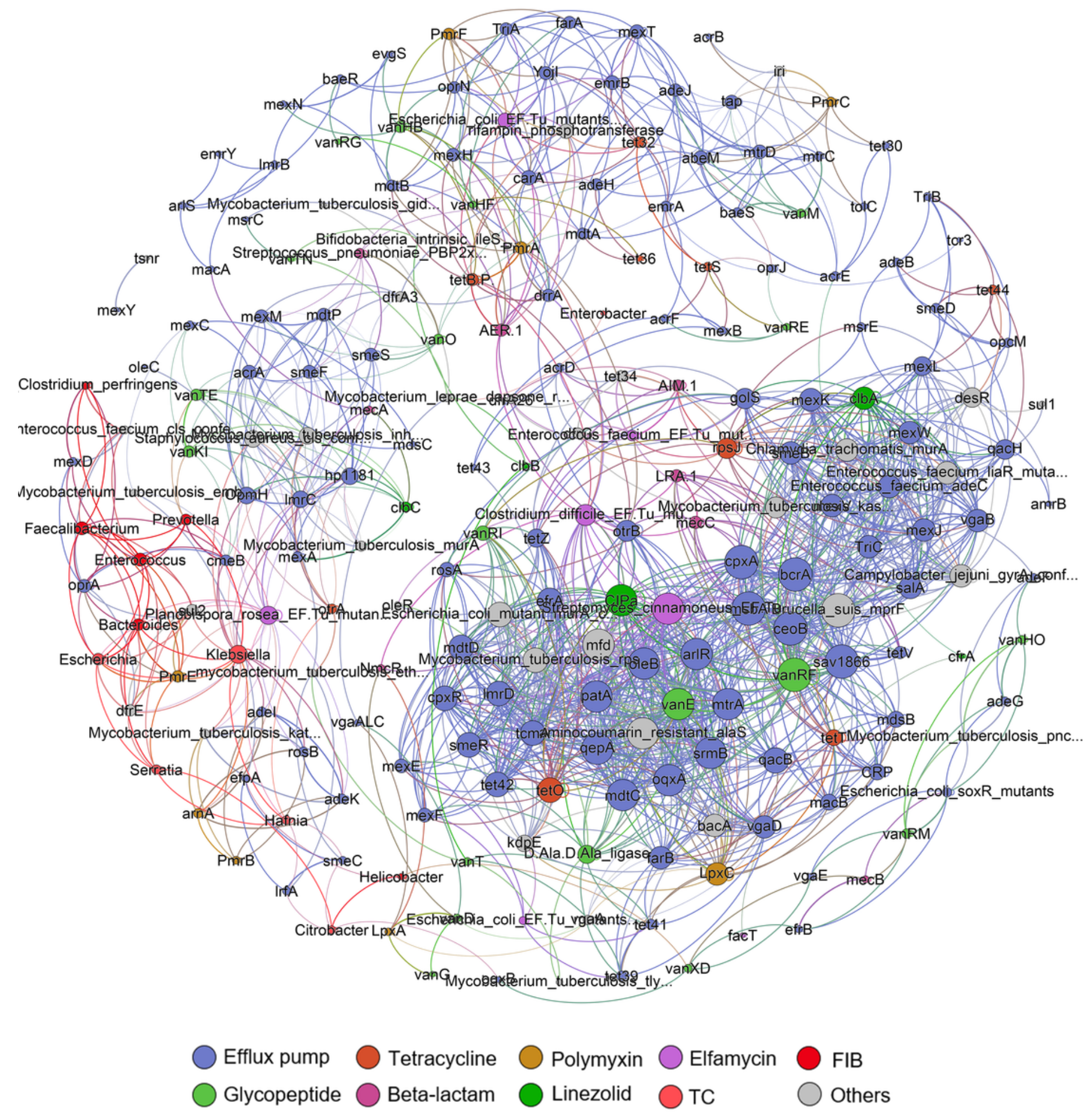

Figure 7

Network analysis reveals the co-occurrence pattern of ARGs and indicator bacteria. Connection represents a strong (Spearman's correlation coefficients $\rho>0.8$, P-value $<0.01$ ) correlation.

\section{Supplementary Files}


This is a list of supplementary files associated with this preprint. Click to download.

- Supplementarydata.docx

- Supplementarytables.xlsx 